\title{
Models as Arguments: An Approach to Computational Science Education
}

\author{
D.E. Stevenson \\ 315 McAdams Hall, School of Computing, Clemson University, \\ PO Box 34097, Clemson, SC 29634-0974 \\ steve@cs.clemson.edu
}

\begin{abstract}
Hardware and software technology have outpaced our ability to develop models and simulations that can utilize them. Furthermore, as models move further into unfamiliar territory, the issues of correctness becomes more difficult to assess. We propose extending classical argumentation structures as the basis for computational science education.
\end{abstract}

\section{Human Centric Computing — The Argument}

Computation and computational science has rightly emphasized hardware and software development. But the hardware is now far more advanced than the software developers can take advantage of and the scientific problems are more complex than those of fifty years ago. Now that computing is pervasive (if not invasive) it is time for humans to catch up. Kurtzweil's "singularity" not withstanding, we must modernize human interaction with computing. The futurists seem to be divided into two camps: pervasive computing and human centric computing (HCC). Once again, however, technology and not people are the focus, with overriding objectives of making the technology transparent or letting each sector focus on what it does best.

- The pervasive computing camp argues that IT needs to "get the end user experience right," focusing on designing devices that are easier for users to interact with in order to find relevant information.

- The objectives of human-centric computing are not to focus on the devices themselves, but rather to create an entire solution so that the human, rather than the device, is always connected.

This is all very exciting: but the human also needs a tune-up, meaning that the education of the 21st Century computational scientist cannot just be more detail at a higher level using the same techniques as those used fifty years ago. So far, the future is about more speed, not about more understanding. The fundamental question before computational science is still "How do we know it's right?"

Since it was fair to test machines for intelligence, machines might test humans with a variant of the Turing test that I will call a Kurzweil Test:

A machine judge engages in a "machine language" conversation with one human and one machine, each of which try to appear as machines. 
All participants are placed in isolated locations. If the judge cannot reliably tell the human from the machine, the human is said to have passed the test. 1

But we are before the singularity, so the Test is to determine if a machine is more intelligent than the human. What do we mean by "more intelligent"? I approach the test by using Bloom's Taxonomy (Fig. 1), which orders cognitive tasks from lowest to highest, as the measure of intellectual achievement and ask questions based on the standard verbs used to identify levels. Now we must ask, "Kurzweil machines will be able to pass the Turing test, will our students pass such a Kurzweil test?"

\begin{tabular}{|l|l|}
\hline Remembering & define, recall, repeat, reproduce state \\
\hline Understanding & classify, explain, identify, translate, paraphrase \\
\hline Applying & choose, demonstrate, interpret, operate, solve, write. \\
\hline Analyzing & appraise criticize, examine, experiment, question, test. \\
\hline Evaluating & defend, judge, select, value, evaluate \\
\hline Creating & construct, create, design, develop, formulate, write. \\
\hline
\end{tabular}

Fig. 1. Bloom's Taxonomy as modified by Lorin Anderson

We can hope that the $\mathrm{Ph}$. D. students can pass a Turing test in their field, but undergraduates probably cannot. Firstly, there is a disconnect between student's abilities to respond to requests to develop artifacts and teachers' expectations. In learning to program, for example, we immediately ask students to create artifacts when their cognitive abilities are still in the lower three categories.

Secondly, the current idea that critical thinking is an ultimate skill to teach leaves us short of creating things: critical thinking is analyzing and evaluating, not creating. In fact, if one looks at classical rhetoric, one finds that critical thinking is a subordinate skill to argumentation.

We extend the concepts presented in 2. Sect. 2 describes computational science problems as we propose they should be solved in a classroom. Sect. 3 discusses a pedagogical approach Systems-Questions-Explanations (SQE). Finally we introduce the addition of argumentation as a pedagogical approach to computational science (Sect. 4) followed by an example (Sect. 5). We collect our major points in Sect. 6.

\section{The Problem}

Computational science in its broadest sense is the modeling of systems. But system models, by themselves, are not necessarily correct. Aristotle was a prolific modeler whose models carried the force of law for almost 2,000 years - but he got the structures of both the universe and biology wrong. Francis Bacon argued in

${ }^{1}$ This tongue-in-cheek version is Wikipedia's explanation of the Turing test [1] with "human" and "machine" swapped. 
his Novum Organon that models must be validated by experiments: the Scientific Method. It is now recognized that the Scientific Method is supported by a third leg: computing. Correctness is an ever more important now than with Bacon and the terms verification and validation are used to describe the processes. Recently, Wilson added reproducibility so we now abbreviate the "correctness" process as VV\&R.

Despite our advanced computer systems, models and simulations are still developed by people. Traditional science and engineering education proceeds by showing the student a number of models, then asking the students to apply these models to textbook problems. This pedagogical approach assumes that students learn to model by exposure. Our data shows that the students are not learning how to create models.

Experiences with incoming science, technology, engineering, and science (STEM) students at Clemson indicates that students do indeed have the facts at hand that we suppose they should have. Many students have high SAT/ACT scores and high predicted GPAs; yet many never complete a STEM degree. One possibility is that these students do not have the critical thinking skills required to analyze scientific models; however, critical thinking skills testing we have conducted (with IRB approval) shows that these students seem to have the critical thinking skills of relatively mature thinkers $[3$. In other words, we have students who are at the evaluting stage in Bloom's Taxonomy but still are not thriving.

The students seem not to understand how to structure an argument, nor are they able to evaluate their own arguments. As discussed in Parham, Chinn, and Stevenson [4, students have trouble solving unstructured problems that have many transitions among the six Bloom elements. By using verbal protocol approach, we were able to trace the students use of information and metacognition. Reviewing, analyzing, and evaluating student or historical arguments is one way to practice critical thinking. We can also practice argumentation by reviewing, analyzing, and evaluating arguments. But argumentation should also require students to structure an argument then review and self-evaluate it [5]. One approach we are trying is to organize modeling classes around classical argumentation methods. Argumentation is both a process (the discussion) and a product (the outcomes) consisting of three parts: rhetoric, reasoning and dialectic. Rhetoric means development of knowledge through communications and dialectic is discovering and testing knowledge through questions and answers. To conduct an orderly argument, we must share standards and appraisal methods relating to knowledge and the reasoning schemata.

\section{How Do Humans Formulate Models? SQE}

The first step in solving any problem is to construct a model of that problem, and this first model is a mental model. The same process of modeling is needed to install new ideas in our personal knowledge bases. The term mental model is used both in formally and informally. Many disciplines have their own interpretations of the term model, but we use three interpretations: (1) model of a physical 
system (physical sciences), (2) models of axioms (logic), and (3) mental models (psychology). The basic interpretation of model is a set of logical (not necessarily classical logic) statements made about the modeled phenomenon that are jointly true - recall that mathematical equations are true or false. The processes for showing models (or simulations) are true are called verification and validation. The "subversive" purpose of this paper is to propose a practical approach to VV\&R education.

While the results of a modeling study are external artifacts, the process of developing models is primarily psychological and sociological. Focusing too much on the external artifacts lends no insight into the thinking process: the reasoning process that education must hone, something quite different than having more theorems and laws. Questions concerning human reasoning are not new, but many modern studies started in the mid-1970s. Philip Johnson-Laird 6] based his concept of mental model on models of explanation put forth by Kenneth Craik 7]. Craik proposed a three step process: (S1) translation of original problem statement to an internal representation, (S2) the manipulation and reasoning to develop a solution, and finally $(\mathrm{S} 3)$ the re-translation of the solution to an external medium.

We discuss S1 and S3 in this section and Step S2 is discussed in 4 Step S1 is a linguistic step, emphasizing two auxiliary steps: association of words with concepts (mind maps) and the association of concepts and relationships (concept maps). At the end of the translation step, the internal representation is a graph of schemata. Schemata are units of knowledge proposed by Immanuel Kant and finally popularized by John Anderson in the $\mathrm{ACT}^{*}$ models. Work on schemata and their use in problem-solving in computer science is being studied at Clemson under the author. Step S3 is interesting in computational science because the output form is usually quite different than the input: complex natural language in, complex computer system out.

\subsection{Psychology}

The author began studying the human aspect of modeling and simulation approximately fifteen years ago, primarily studying problem-solving as described by Sternberg [8]. There are five aspects of problem solving: modeling (planning), reasoning, knowledge, critical thinking, and creative thinking. Bushey [3] explored the role of critical thinking, showing that successful computer scientists are critical thinkers. As research continued into the psychology literature, we soon focused on the issue of knowledge and how it is used.

Anderson [9] proposed the first of a series of model 2 collectively called ACT*, based on the concept that humans hold knowledge in certain memories, that these units of memories are connected, and that we use pattern-matching to find new connections. The dynamics of how this might occur were partially established by Marshall [10]. While Marshall established some evidence for schemata, she did not establish how we recognize mistakes. This process is now known

$\overline{2}$ These models are an active domain of research in cognitive psychology. 
as metacognition, first introduced by Flavell [11]. The emerging picture is that the problem solver recognizes patterns of information that identifies schemata and their connections with metacognition playing a control function. Schemata themselves seem to be units of knowledge with ability to determine applicability, planning, and execution requirements.

\section{Systems, Questions, and Explanations}

This is still an unsatisfactory explanation of how humans learn to be modelers: how did Einstein happen on his elevator? Our goal is to understand the tools needed to model a system and inculcate them into the students. This was discussed in Stevenson [2], which proposes that the modeling is based on three foci: Systems, Questions, and Explanations (SQE). The reasoning for the SQE model is as follows: In order to ask a meaningful question, one must first have a system in mind. The system effectively defines the context, rules, and conditions that the system obeys and provides a vocabulary of variables and values (at resolution) to provide semantics for both the question and the answer. With the system and question, one can provide meaningful answers, which themselves must be explained; justification is just one aspect of explanation.

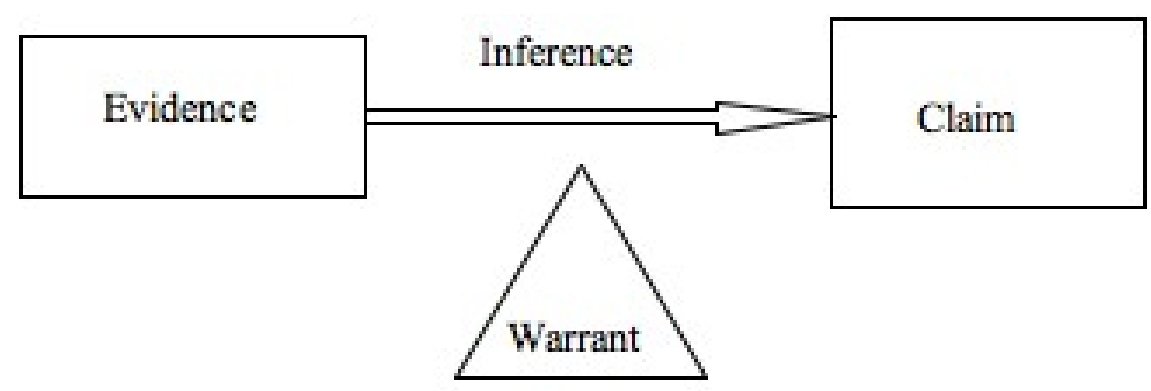

Fig. 2. Simplified Unit Argumentation Cell. Four fundamental information types. Claims are the conclusion. Evidence supports claims through inference. Inference is justified by warrants.

The development of an argument relies on the structure shown in Fig. 2 , Classically, these parts are logical statements playing four roles:

1. Claim. This is the statement to be the conclusion.

2. Inference. A gap can be filled by inference based on the structure of the model. These inferences must be justified (warranted).

3. Evidence and Hypotheses. A gap may be filled by hypotheses that must then be proven.

4. Computation. A gap may imply enough conditions that a computation is feasible and practical. 
This particular approach comes from a basic observation: one must argue that a model is correct and therefore the model is the argument. This model accepts the closed world view. In the open world models, there are defaults and uncontrolled logical situations; in the closed world, all propositions must be expressed explicitly. There are fine lines of distinction between claims, evidence, inference, and warrants that the student must wrestle with, but it is a clear framework.

Finally, the work on mental models 1213 shows that most people use informal methods of reasoning [5]. Evidence indicates that we reason through logical models, but not necessarily using classical (simple syllogistic) logic. One approach, for example, might be to integrate new information in the older model, then deal with the contradictions and incoherencies. The basic problem is that classical logical deduction only works if we have all the facts and we have a set of axioms and we have a set of rules of inference. By definition, as we learn we do not have those things - they're a work in progress. This particular issue is now known as "informal logic".

\subsection{Argumentation}

Classically, there are three parts to discourse: logic, dialectic (discussing the truth of opinions) and rhetoric. A major theme in rhetoric is argumentation, including debates. [Note: There is no way to do justice to argumentation in such a short paper. We present a very short overview]. Arguments are decision-making processes that is carried out in an uncertain environment. An argument consists of four elements: claims, evidence, inference, and warrants, themselves logical statements. Claims are the conclusion of an argument and are not necessarily universal truths and hence the participants values, priorities, and methods of judgment are crucial. In order to arrive at a comon ground, the participants must adopt

- A critical thinking regimen,

- A common language and semantics,

- Procedural assumptions and norms

- A common frame or frames of reference

Fundamental reasoning skills are essential. The Unit Cell (Fig. 2) is effectively the rationale for accepting the claim when an inferential step should be accepted. Conclusions (claims) must be justified (warranted). Unlike formal syntactic justifications, arguments can be subjective [14, because knowledge can also be subjective. The adoption of skepticism is required, hence critical thinking is a key component. Knowledge has degrees of strength and is always provisional: our understanding could change with a simple experiment.

How can we use this for 21st Century education? The subject provides a framework to consider the development of a field. The following classical subjects form such a framework [15]16]:

- Evaluating Evidence. This is the study of both good and bad arguments. This is similar to requiring students to look at models and deciding whether they believe the evidence. The first third of [17] emphasizes these skills. 
- Understanding the Issues. Students often do not know what the issue is when it comes to analyzing a problem. Most problems have a limited number of issues that must be addressed, but those issues must be apparent to the students.

- Case Construction. Case construction is the heart of argumentation, by this I mean choosing the unit cells (Fig. 2)you wish to use. Each subject has a limited number of standard arguments that any competent practioner understands.

- Inferences and Warrants. While most academic subjects try to use classical logic (But not intuitionistic logic ... grist for another article), the psychological studies of human reasoning indicate humans do not naturally do so. Formulating the statement is not the same as warranting (justifying) the statement. Physics principles such as Conservation of Energy laws fit in this area.

- Fallacious Reasoning. Fallacies take up a large portion of argumentation classes, but it is not clear how much time should be taken up in "applied" classes. Some disciplines such as mathematics have famous fallacies (the pons asinorum, for example).

Fortunately there are already examples of such approaches in computational science.

Test Driven Development (TDD). This is a method of developing software that adopts a test-first approach. Instead of writing a complete program first and then testing the program once it has been developed, TDD has the software programmers constantly testing the software from the first step. Tests are created for most or all of the individual elements of a program, such as the functions, objects, etc.

Model-Based Techniques. Model-based testing is closely related to modelbased specification. Models are used to describe the behavior of the system under consideration and to guide such efforts as test selection and test results evaluation. Both testing and verification are used to validate models against the requirements and check that the implementation conforms to the specification model. Of particular importance are formal models with precise semantics, such as state-based formalisms. Techniques to support model-based testing are drawn from diverse areas, like formal verification, model checking, control and data flow analysis, grammar analysis, and Markov decision processes.

Patterns as Schemata. The formal descriptions of patterns, as in [18, are attempts to describe successful solutions to common problems. Patterns exist across the knowledge spectrum, in all disciplines. Software patterns are a type of schemata. Educating students to recognize patterns helps connect them with their own knowledge rather than just resolving a problem. Not only do patterns teach useful techniques, they help people communicate better, and they help people reason about what they do and why. 
This is not very mystical: electrical engineering circuit diagrams are a type of pattern. Recognition of patterns as decomposition tools in problem solving is a hallmark of expertise. A person's collection of schemata concerning a problem form a graph representing that person's knowledge: a language about a particular program providing vocabulary for talking about a particular problem. Patterns have a context in which they apply.

\section{An Example}

An example of how this approach can be used actually occurred recently in one of my classes. This class has a semester-long project with seven milestones. Starting with Milestone 2, the students must test their own code before I do. One would think that seniors know how to think about testing such a project - unfortunately, ours do not. Therefore, the problem to be solved is "how to thinking about testing."

What is a model to the problem at hand? They want to claim that they have completely tested their code. I drew the unit cell on the board and labeled it. I then asked the students (I use problem-based learning exclusively), "What do you want to claim?" It took some time for them to respond, "We want you to accept our milestones as correct."

"What is your evidence"?

This took considerably longer before they could formulate that the evidence was actually four separate classes of processing. The project they were working on requires them to accept input or reject it. While they finally determined that there were four classes of input to demonstrate, they never did determine that they also had to show they should reject some inputs. When I pointed that out, we have eight separate types of test inputs.

"Why should I believe you"?

Again, this took some discussion, but we finally agreed that they were presenting direct evidence and could map the specification onto the inputs and outputs.

\section{Conclusions}

Early versions of this paper were laid out in a tableau format to simplify the task of organizing the material. An interesting observation is that the tableau is almost exactly what Euclid taught in the Elements. The connection is important, but our main message is this:

- To develop the best in our students they must reach the "creating" level of Bloom's Taxonomy as quickly as possible.

- To be at the creating level means that both critical thinking and argumentation (modeling) must be emphasized.

- Modeling begins with mental modeling.

- Modeling is an inductive process.

Final Comment. It may seem odd that there is little mention of reasoning and logic. There are several reasons for this, primarily the fact that any discussion of these subjects is a paper unto itself. 


\section{Acknowledgement}

The author wishes to acknowledge the discussions with Dr. Donald Chinn, Ms. Jennifer Parham and the entire group working with the First Generation College Students at Clemson: Drs. Barbara Speziale, Jeffrey Appling, Calvin Williams, Robert Ballard, Matthew Ohland, and John Wagner. Ms. Sherry Dorris provided insights on how students were reacting to certain subjects. The students in my Honors class on "Understanding Scientific Reasoning" were most helpful by providing feedback and discussing how they actually study (or don't).

\section{References}

1. Turing, A.: Computing machinery and intelligence. Mind LIX (Oct. 1950) 433-460 doi:10.1093/mind/LIX.236.433.

2. Stevenson, D.E.: The problem with problems in computational science and engineering problem-based learning. In: 2006 International Conference on Computational Science and Education. (Aug 2006)

3. Bushey, D.E.: Critical thinking traits of top-tier experts and implications for computer science education. $\mathrm{PhD}$ thesis, Clemson University, Clemson, SC (August 2007)

4. Parham, J., Chinn, D., Stevenson, D.E.: Using bloom's taxonomy to code verbal protocols of students solving a data structure problem. In: Proceedings of ACM SE 2009. (2009) Submitted.

5. Walton, D.: Informal Logic: A Pragmatic Approach. Cambridge University Press, Cambridge (2008)

6. Johnson-Laird, P.: Mental Models. Harvard University Press (1983)

7. Craik, K.: The Nature of Explanation. Cambridge University Press, Cambridge, England (1943)

8. Davidson, J.E., Sternberg, R.J.: The Psychology of Problem Solving. Cambridge University Press, Cambridge, UK (2003)

9. Anderson, J.: Language, Memory, and Thought. Erlbaum (1976)

10. Marshall, S.P.: Schemas in Problem Solving. Cambridge University Press (1995)

11. Flavell, J.H.: Metacognitive aspects of problem solving. In Resnick, L.B., ed.: The Nature of Intelligence. Erlbaum, Hillsdale, NJ (1976) 231-236

12. Johnson-Laird, P.: How We Reason. Oxford University Press (2009)

13. Stenning, K., van Lambalgen, M.: Human Reasoning and Cognitive Science. Bradford Books (2008)

14. Walton, D.: Fundamentals of Critical Argumentation (Critical Reasoning and Argumentation. Cambridge University Press (2005)

15. Freeley, A.J., Steinberg, D.L.: Argumentation and Debate: Critical Thinking for Reasoned Decision Making. 10th edn. Wadsworth (1999)

16. Zarefsky, D.: Argumentation: The Study of Effective Reasoning. The Thinking Company (2005)

17. Giere, R.N., Bickle, J., Mauldin, R.: Understanding Scientific Reasoning. Wadsworth (2005)

18. Mattson, T.G., Sanders, B.A., Massingill, B.L.: Patterns for Parallel Programming. Addison-Wesley (2005) 